\title{
Dinámica a largo plazo de las praderas de carófitos y los masegares en el humedal de Las Tablas de Daimiel (Ciudad Real, España): relación con las perturbaciones ambientales
}

\author{
S. CIRUJANO ${ }^{1}$, N. GUERRERO² \& M. ÁLVAREZ COBELAS ${ }^{3}$ \\ ${ }^{1}$ Real Jardín Botánico, CSIC, pl. de Murillo, 2, ES-28014 Madrid, España \\ ${ }^{2}$ EINTAM Estudios Europeos de Medio Ambiente, S.L., c. Juan Ramón Jiménez, 28, $3^{\circ} \mathrm{D}$, ES-28036 Madrid, España \\ ${ }^{3}$ Museo Nacional de Ciencias Naturales, CSIC, c. José Gutiérrez Abascal, 2, ES-28006 Madrid, España \\ Trabajo dedicado al doctor Francisco Javier Fernández Casas con motivo de su jubilación
}

ORCID iD. S. CIRUJANO: http://orcid.org/0000-0003-4979-6735, N. GUERRERO: http://orcid.org/0000-0003-4709-1758, M. ÁLVAREZ COBELAS: http://orcid.org/0000-0001-5479-0624

Autor para correspondencia: S. Cirujano (santos@rjb.csic.es)

Editores: N. Ibáñez \& J. López-Pujol

Recibido 24 octubre 2016; aceptado 25 enero 2017; publicado on line 10 mayo 2017

\begin{abstract}
LONG TERM DYNAMics OF CHAROPHYTES MEADOWS AND CUT-SEDGE BEDS IN THE WETLAND OF LAS TABlas de Daimiel (CIUdAD Real, Spain): Relationship with ENVIRONMENTAL Disturbances.- This work analyses surface variations on charophyte meadows and cut-sedge beds in Las Tablas de Daimiel National Park (Ciudad Real, Spain) from the 1950s to the present, and its relationship with the changes that have occurred in the wetland meanwhile (e.g. droughts, fires, pollution episodes). Therefore, the role of these two parameters as environmental indicators at this continental wetland has been assessed.
\end{abstract}

Key words: aquatic macrophytes; Charophytes; Cladium mariscus; EU Water Framework Directive; Las Tablas de Daimiel National Park; Mediterranean floodplains.

\section{Resumen}

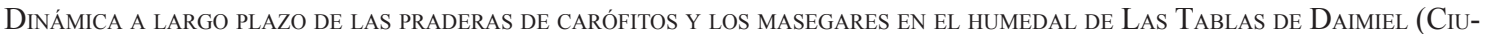
DAD REAL, España): RELACIÓN CON LAS PERTURBACIONES AMBIENTALES. - Este trabajo presenta los cambios en la extensión de las superficies ocupadas por las praderas sumergidas de carófitos y por las formaciones emergentes de masiega en el humedal del Parque Nacional Las Tablas de Daimiel (Ciudad Real, España). Se estudian las dinámicas de ocupación desde los años cincuenta hasta la actualidad, y se relacionan con las perturbaciones más relevantes como sequía, incendios y contaminación. Se destaca el papel de estos dos componentes del ecosistema acuático como indicadores de la calidad ambiental de esta zona húmeda continental.

Palabras clave: Cladium mariscus; Characeae; Directiva Marco del Agua; humedales mediterráneos; macrófitos acuáticos; Parque Nacional Las Tablas de Daimiel.

\section{Cómo citar este artículo / Citation}

Cirujano, S., Guerrero, N. \& Álvarez Cobelas, M. 2017. Dinámica a largo plazo de las praderas de carófitos y los masegares en el humedal de Las Tablas de Daimiel (Ciudad Real, España): relación con las perturbaciones ambientales. Collectanea Botanica 36: e005. doi: http://dx.doi.org/10.3989/collectbot.2017.v36.005

\section{Copyright}

(C) 2017 CSIC. This is an open-access article distributed under the terms of the Creative Commons Attribution License (CC BY) Spain 3.0. 


\section{INTRODUCCIÓN}

El Parque Nacional Las Tablas de Daimiel, con una extensión actual de 3300 ha, es una llanura que cuenta con unas 1600 ha de superficie inundable situada al final de una amplia cuenca hidrográfica de $15.000 \mathrm{~km}^{2}$ (Fig. 1). Lo que ocurre en la cuenca tiene su repercusión directa o indirectamente en el humedal: sobreexplotación de las aguas subterráneas, vertidos, contaminación agrícola difusa, entrada de fauna alóctona, etc. A estos impactos hay que añadir los ocurridos en el propio parque: intentos de desecación, eliminación de los masegares, incendios, proliferación de especies invasoras, etc. (Sánchez Carrillo \& Angeler, 2010).
E1 Parque Nacional Las Tablas de Daimiel constituye uno de los ecosistemas más peculiares de la península ibérica. Esta llanura de inundación estaba alimentada esencialmente por el desbordamiento de dos ríos y de manantiales u «ojos» (Álvarez Cobelas \& Cirujano, 1996; Domínguez et al., 2006; Sánchez Carrillo \& Angeler, 2010). Los ríos eran de distinta naturaleza, el Guadiana, de aguas bicarbonatadas y permanentes, y el Gigüela, de aguas sulfatadas y estacionales; los manantiales son surgencias de aguas freáticas de la llamada Masa de Agua Mancha Occidental I y II (antes Acuífero 23 o Unidad Hidrogeológica 04.04).

Si bien Las Tablas de Daimiel eran en origen un humedal semipermanente con una inundación

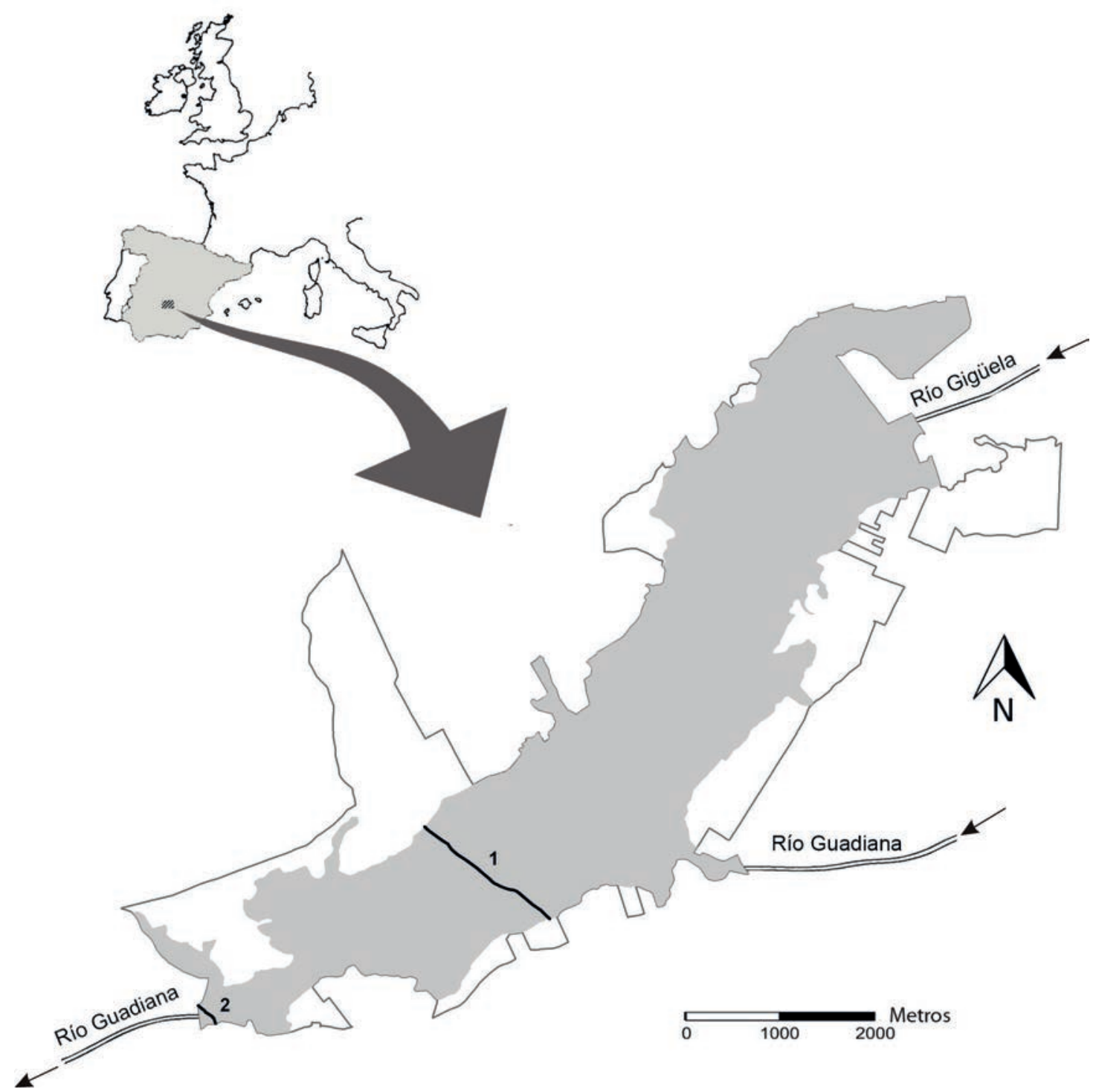

Figura 1. Localización y límites del Parque Nacional Las Tablas de Daimiel. En color gris la superficie potencialmente inundable. 1: presa central; 2: presa de Puente Navarro. 
máxima a comienzos de la primavera, y disminuciones paulatinas posteriores sin llegar al estiaje - ya que los aportes del río Guadiana eran permanentes y mantenían unas 500-600 ha inundadas durante todo el año-, hoy se han convertido en una zona húmeda muy fluctuante, con periodos de inundación máxima y otros de estiaje prácticamente total, a lo que ha contribuido el cese de los aportes del mencionado río desde aproximadamente el año 1986 hasta 2012, cuando volvió a entrar agua procedente de dicho cauce. Además, las dos presas construidas en la década de 1980, una dentro del Parque y otra al final del mismo, también han contribuido a modificar la hidrología del sistema (Figs. 1 y 2 ).

Junto con unos aportes hídricos fluctuantes, la contaminación del agua embalsada en Las Tablas ha sido un problema recurrente, especialmente después de su declaración como Parque Nacional en 1973 (Fig. 2). La contaminación difusa que llega al humedal, que procede fundamentalmente del uso agrícola de una gran parte de los aproximadamente 15.000 $\mathrm{km}^{2}$ de la cuenca hidrográfica receptora, y los episodios puntuales de contaminación aguda, procedentes de diversos núcleos urbanos e industriales, han aportado una carga excesiva de nutrientes y contaminantes que han contribuido a la eutrofización del sistema y a potenciar sus efectos ambientales. Además del impacto de estos dos factores, no hay que olvidar la presión ejercida por la fauna acuática introducida. Más información sobre la hidrología, el ambiente físico-químico, la biodiversidad y la ecología de este ecosistema singular se puede encontrar compendiada en Álvarez Cobelas \& Cirujano (1996) y Sánchez Carrillo \& Angeler (2010).

La Directiva 2000/60/CE o Directiva Marco del Agua, en vigor desde 2000 (DOCE, 2000), establece una serie de indicadores biológicos que permiten determinar el estado ecológico de las masas de agua. Entre estos indicadores se contempla la abundancia y composición de los macrófitos acuáticos, entendiéndose como tales las plantas que se ven a simple vista y que pueden clasificarse como hidrófitos, helófitos e higrófitos (Den Hartog \& Segal, 1964; Cirujano et al., 2007).

Los carófitos son un grupo de hidrófitos sumergidos, pertenecientes a la familia Characeae, división Chlorophyta, que al quedar en contacto con el aire se secan y mueren. Algunas especies o variedades de este grupo de algas son más sensibles que otras a la contaminación, pero todas terminan por extinguirse cuando la eutrofia del agua aumenta y se manifiestan los efectos asociados al incremento de nutrientes (Blindow, 1992; Van den Berg et al., 1998; Coops, 2002; Cirujano et al., 2007; Rodrigo et al., 2010; Alonso Guillén, 2011).

La masiega [Cladium mariscus (L.) Pohl] es un helófito, de la familia Cyperaceae (Cirujano et al., 2014), que en la península ibérica es característica de lagunas y humedales con aguas ricas en carbonatos-bicarbonatos. En estos ecosistemas la masiega ocupa bandas de anchura variable que, según la morfología del cuerpo de agua, pueden constituir formaciones impenetrables denominadas masegares

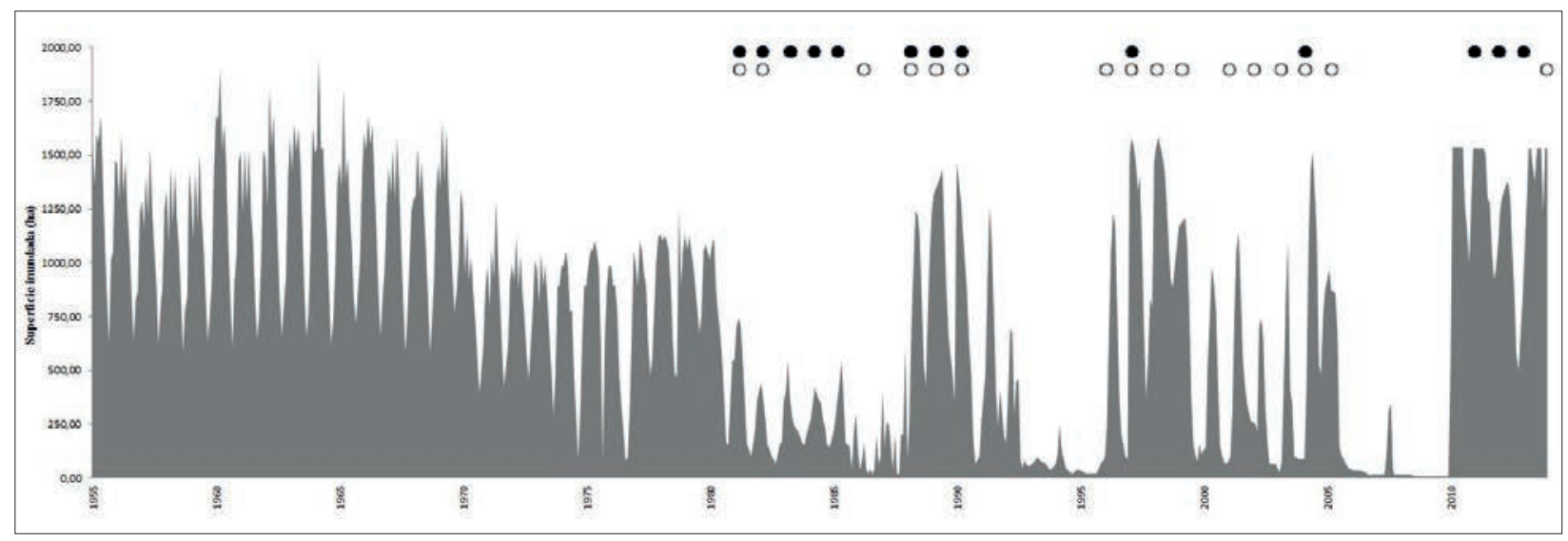

Figura 2. Evolución de los niveles de inundación en el Parque Nacional Las Tablas de Daimiel entre los años 1955 y 2014, y los principales episodios de contaminación ocurridos desde 1981 hasta 2014. •: episodios de contaminación aguda; ○: episodios de contaminación difusa. Fuente: publicaciones, informes encargados por diversos organismos públicos (ICONA, Organismo Autónomo Parques Nacionales) y comunicación oral de la dirección del Parque (INYPSA, 1990; Álvarez Cobelas \& Cirujano, 1996, 1998, 2006, 2010, 2011, 2012 y 2015). 
(Conway, 1936, 1937, 1938a, b; Cirujano \& Medina, 2002; Ribeiro, 2004).

El objetivo de este trabajo es demostrar que la extensión de las formaciones subacuáticas de carófitos puede elegirse como indicador a corto plazo del buen estado de conservación del ecosistema, ya que su desarrollo está condicionado por la calidad del agua, por la duración anual de la inundación $\mathrm{y}$ por el efecto que sobre ella ejerce de la fauna acuática introducida. Además, la masiega, puede emplearse como indicador a medio y largo plazo de los cambios ocurridos en el ecosistema; sobre todo, los que se refieren a la duración prolongada de la fase seca y a las modificaciones en la tipología iónica del agua.

\section{MATERIAL Y MÉTODOS}

La evolución anual de la superficie de inundación ha sido recopilada por la dirección del Parque $\mathrm{Na}-$ cional desde 1989 hasta la actualidad. Los datos iniciales desde 1955 fueron interpolados por regresión lineal múltiple (Álvarez Cobelas et al., 2001).

El análisis de las superficies colonizadas por las formaciones subacuáticas de carófitos y las formaciones de masiega se realizó con los mapas de vegetación disponibles a partir del año 1956. Los mapas del 1956 al 1984 se elaboraron con la fotografía convencional y aérea disponible de dichos años, y los datos aportados por los antiguos habitantes de Las Tablas (Álvarez Cobelas \& Cirujano, 1996, 2015). El resto de los mapas desde 1993 a 2014 se basan igualmente en fotografías aéreas restituidas a ortofotografías y en las observaciones y muestreos efectuados en el humedal. Para cada uno de estos años, se delimitó la extensión de los diferentes tipos de formaciones vegetales: vegetación subacuática, emergente y marginal, praderas juncales, bosquetes de tarayes, juncales, etc. (Álvarez Cobelas \& Cirujano, 1996, 1998, 2006, 2010, 2011, 2012).

Los cálculos para determinar la cobertura de las praderas subacuáticas de carófitos se realizaron en el mes de junio, periodo en el que dichas formaciones están bien constituidas. La superficie inundada en dicha fecha, que no estaba ocupada por la vegetación emergente, se estimó como la que potencialmente podía ser colonizada por los carófitos. La superficie real ocupada por estas formaciones se precisó mediante muestreos realizados por toda la superficie inundada en el periodo 1992-2014. En los años anteriores 1956, 1977 y 1984 se consideró, dado que la calidad del agua era adecuada, que la superficie real coincidía con la potencialmente colonizable.

La extensión de los masegares se estimó discriminando en la vegetación emergente entre dichas formaciones y las de carrizo [Phragmites australis (Cav.) Trin. ex Steud.] y enea [Typha domingensis (Pers.) Steud.]. Estas tres especies son los helófitos más característicos del humedal.

\section{RESULTADOS Y DISCUSIÓN}

Las praderas subacuáticas de carófitos ocupaban en el año 1956, cuando Las Tablas estaban inalteradas, unas 450 ha que era prácticamente el $100 \%$ de la superficie que podían ocupar al estar libre de vegetación emergente (Fig. 3). Durante los años 70 y 80 se acentuó la pérdida de aportes debido a la sobreexplotación de las aguas subterráneas y la entrada de aguas contaminadas. En el año 1984 la superficie máxima inundada fue de 419 ha, y la del mes de junio de 361 ha, con una cobertura de carófitos de 125 ha. En el año 1987 se puso en marcha el denominado Plan de Regeneración Hídrica que permitió la derivación, a partir del año 1989, de diferentes volúmenes de agua hacia Las Tablas procedentes del trasvase Tajo-Segura. Dicho Plan tuvo en cuenta la cantidad de agua a trasvasar pero no su calidad (Álvarez Cobelas \& Cirujano, 1996).

Las diferencias entre las superficies potencialmente colonizables por las praderas de carófitos y las realmente colonizadas son notorias a partir del año 1993. Esto se acentuó aún más en el año 2005, porque la contaminación aguda ocurrida en el año 2004 (Fig. 2) arrasó casi por completo la vegetación subacuática, que ya no se recuperó debido a la conjunción de un periodo seco que duró hasta el año 2010 (Álvarez Cobelas \& Cirujano, 2006, 2010).

A este periodo, que se prolongó desde 2007 hasta finales de 2010 en el que Las Tablas quedaron completamente secas, sucedió una fase húmeda con inundaciones máximas hasta el año 2014. Tras la eliminación en el año 2009 de unas 18.000 tm de vegetación anual y nitrófila, tarayares de escaso porte [Conyza canadensis (L.) Cronq., Conyza bonariensis (L.) Cronq., Aster squamatus (Spreng.) 


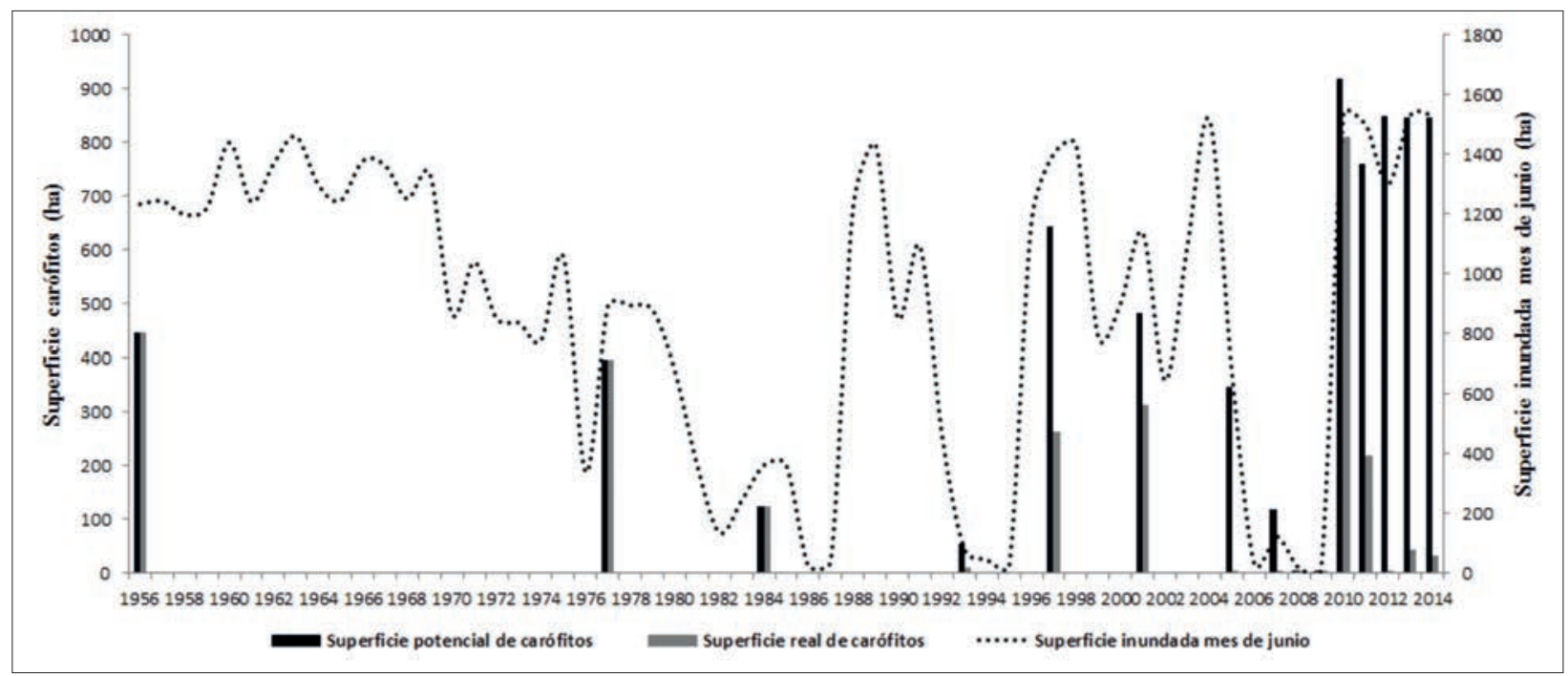

Figura 3. Comparación de las superficies potencial y real colonizadas por los carófitos en Las Tablas de Daimiel. Datos correspondientes al mes de junio de diferentes años desde 1956 a 2014. Se indica también la dinámica de la superficie inundada en dicho mes.

Hieron., Cochlearia glastifolia L., Tamarix canariensis Willd.] y helofítica (Phragmites australis) la superficie libre de vegetación e inundada en el mes de junio llegó a las 920 ha, de las cuales unas 811 ha fueron colonizadas por las praderas subacuáticas de carófitos (Fig. 3), lo que supone casi el doble de la superficie existente en el año 1956 (Álvarez Cobelas \& Cirujano, 2010).

Las entradas de vertidos contaminantes por el río Gigüela y por el antiguo cauce del Guadiana se iniciaron en el verano de 2010 y se prolongaron hasta 2014 (Fig. 2). Esta situación, unida al impacto causado por la excesiva población piscícola, incluida la que genera gran turbidez en el agua, ha contribuido a que en el periodo 2011 a 2014 la superficie cubierta por las praderas de carófitos no alcance ni el $4 \%$ de la superficie potencialmente colonizable (Fig. 3) (Álvarez Cobelas \& Cirujano, 2011, 2012; Laguna et al., 2016).

La superficie cubierta por los masegares de Cladium mariscus en el año 1956 era de unas 1078 ha (Fig. 4). En la década de 1970 se desecaron y pusieron en cultivo unas 410 ha, situadas entre la presa central y la de Puente Navarro (Fig. 1), reduciéndose un $38 \%$ la superficie colonizada por esta formación, que pasó a ocupar 669 ha en 1977. El incendio de turba ocurrido en el año 1986 calcinó otra parte del masegar situado en la zona central del parque (Álvarez Cobelas \& Cirujano, 1996), entre la mencionada presa central y la entrada del río Guadiana
(Fig. 1), y en el año 1993 los masegares ocuparon 405 ha, el $62 \%$ menos que en 1956 . Esta tendencia decreciente de la superficie ocupada por los masegares se mantuvo hasta 2009, año extremadamente seco en el que se produjo una nueva combustión de la turba de la zona central, y descendió a 96 ha, lo que supone una disminución del $91 \%$ frente a la de 1956. Tras la inundación máxima ocurrida en el año 2010, que se ha prolongado hasta la actualidad, los masegares no se recuperaron, ya que después de estar sometidos a un fuerte estrés hídrico, sucedió la inundación máxima que incluso los cubrió en algunas zonas (Ortiz Llorente, 2013). Finalmente, en el periodo 2010-2014 la superficie cubierta por los masegares ha quedado reducida a unas 15-16 ha, lo que significa que su superficie se ha reducido un $98 \%$ respecto a la que ocuparon en el año 1956 (Álvarez Cobelas \& Cirujano, 2012).

Como se ha visto, los cambios en la distribución de los carófitos y masegares se relacionan bien con fuertes perturbaciones en el humedal y vienen a corroborar la relación de la pérdida de diversidad y la extensión de las formaciones vegetales con los cambios acaecidos al medio acuático durante las últimas décadas. Las perturbaciones tienen su origen principalmente en: (1) los intentos de desecación que tuvieron lugar entre los años 1967 y 1973; (2) la alteración del régimen hídrico original derivado de la sobreexplotación de las aguas subterráneas con fines agrícolas; (3) los incendios de 


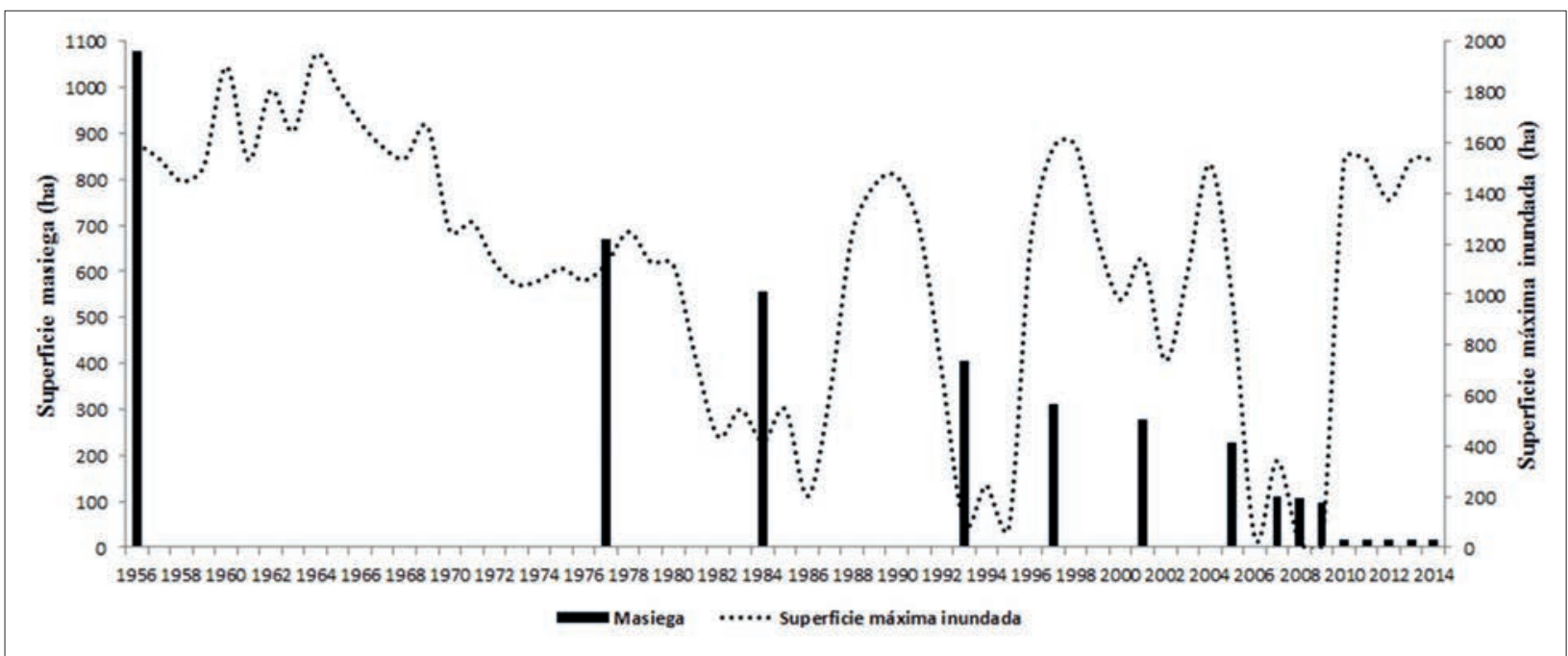

Figura 4. Cambios en las superficies de los masegares en el periodo 1956 a 2014, y evolución de la superficie máxima inundada en Las Tablas de Daimiel.

diferente intensidad ocurridos en los años 1986, 1987, 1994 y 2009; (4) la contaminación del agua por sucesivos y reiterados vertidos de aguas residuales urbanas e industriales; (5) el aumento de la turbidez del agua derivado de la ausencia de vegetación sumergida; (6) la proliferación excesiva de especies introducidas consumidoras de este tipo de vegetación, como son carpas (Cyprinus carpio Linnaeus, 1758), carpines (Carassius carassius Linnaeus, 1758), cangrejo americano (Procambarus clarkii Girard, 1852) y, desde 2010, el pez gato (Ameiurus melas Rafinesque, 1820) (Álvarez Cobelas \& Cirujano, 1996; Angeler et al., 2002, 2003; Cirujano et al., 2004; Sánchez Carrillo \& Angeler, 2010; Laguna et al., 2016).

\section{CONCLUSIONES}

Las praderas subacuáticas de carófitos ponen de manifiesto que aún en periodos de bonanza hídrica no logran regenerarse, debido a la deficiente calidad de las aguas embalsadas y la presión de la fauna exótica. El claro desfase que se produce entre la superficie teóricamente colonizable y la colonizada evidencia estas afecciones.

Los masegares, uno de los elementos característicos del humedal, han pasado de ser la vegetación emergente dominante en el humedal, a un elemento testimonial con una reducción de la superficie colonizada del $98 \%$ respecto al año 1956 . También son un claro exponente de los cambios ocurridos y de cómo el sistema se mueve hacia otro modelo más banal de zona húmeda.

Por tanto, las formaciones acuáticas de carófitos como indicador anual de la calidad y la permanencia del agua, y los masegares como indicador a medio y largo plazo de los cambios en la hidrología, muestran lo que ha ocurrido y sigue ocurriendo en este Parque Nacional.

\section{AGRADECIMIENTOS}

La mayor parte de los datos utilizados para elaborar el presente artículo están extraídos de diferentes proyectos de investigación y de seguimiento financiados por el Organismo Autónomo Parques Nacionales.

\section{REFERENCIAS BIBLIOGRÁFICAS}

Alonso Guillén, J. L. 2011. Los carófitos en la restauración de ecosistemas acuáticos. Un caso práctico en el Parc Natural de l'Albufera de València. Tesis Doctoral, Institut Cavanilles de Biodiversitat i Biologia Evolutiva, Universitat de València, Valencia.

Álvarez Cobelas, M. \& Cirujano, S. (Eds.) 1996. Las Tablas de Daimiel: Ecología acuática y sociedad. Organismo Autónomo Parques Nacionales, Madrid.

Álvarez Cobelas, M. \& Cirujano, S. (Coords.) 1998. Estudio limnológico y botánico del Parque Nacional Las Tablas de Daimiel. Organismo Autónomo Parques Nacionales, Madrid.

Álvarez Cobelas, M. \& Cirujano, S. (Coords.) 2006. Seguimiento y monitoreo ambiental en el P.N. Las Tablas de Daimiel. 2003-2005. Organismo Autónomo Parques Nacionales, Madrid. 
Álvarez Cobelas, M. \& Cirujano, S. (Coords.) 2010. Asesoramiento ambiental a la Dirección del Parque y diseño de actuaciones para la recuperación ambiental gradual de Las Tablas de Daimiel. 2008-2010. Organismo Autónomo Parques Nacionales, Madrid.

Álvarez Cobelas, M. \& Cirujano, S. (Coords.) 2011. Seguimiento ambiental del Parque Nacional Las Tablas de Daimiel (Ciudad Real). Organismo Autónomo Parques Nacionales, Madrid.

Álvarez Cobelas, M. \& Cirujano, S. (Coords.) 2012. Seguimiento ambiental del Parque Nacional Las Tablas de Daimiel (Ciudad Real). Organismo Autónomo Parques Nacionales, Madrid.

Álvarez Cobelas, M., \& Cirujano, S. (Eds.) 2015. Flor Ribera. La gente del río en La Mancha (Limonoiberia, 3): Grupo de Investigación del Agua, Madrid.

Álvarez Cobelas, M., Cirujano, S. \& Sánchez Carrillo, S. 2001. Hydrological and botanical man-made changes in the Spanish wetland of Las Tablas de Daimiel. Biological Conservation 97: 89-98. https://doi.org/10.1016/S00063207(00)00102-6

Angeler, D. G., Álvarez Cobelas, M., Sánchez Carrillo, S. \& Rodrigo, M. A. 2002. Assessment of exotic fish impacts on water quality and zooplankton in a degraded semi-arid floodplain wetland. Aquatic Sciences 64: 76-86. https://doi. org/10.1007/s00027-002-8056-y

Angeler, D. G., Sánchez-Carrillo, S., Álvarez Cobelas, M., Cirujano, S. \& Medina. L. 2003. Exotic crayfish activity and its effects on water quality: preliminary implications for the alternative stable equilibria in Mediterranean wetlands. Journal of Mediterranean Ecology 4: 13-21.

Blindow, I. 1992. Decline of charophytes during eutrophication: comparison with angiosperms. Freshwater Biology 1: 15-27. https://doi.org/10.1111/j.1365-2427.1992. tb00558.x

Cirujano, S. \& Medina, L. 2002. Plantas acuáticas de las lagunas y humedales de Castilla-La Mancha. Real Jardín Botánico (CSIC), Madrid \& Junta de Comunidades de CastillaLa Mancha, Toledo.

Cirujano, S., Camargo, J. \& Gómez-Cordovés, C. 2004. Feeding preference of the red swamp crayfish Procambarus clarkii (Girard) on living macrophytes in a Spanish Wetland. Journal of Freshwater Ecology 19: 219-225. https:// doi.org/10.1080/02705060.2004.9664535

Cirujano, S., Cambra, J., Sánchez Castillo, P. M., Meco, A. \& Flor Arnau, N. 2007. Flora ibérica. Algas continentales. Carófitos (Characeae). Real Jardín Botánico (CSIC), Madrid.

Cirujano, S., Meco, A., García Murillo, P. \& Chirino, M. 2014. Flora acuática española. Hidrófitos vasculares. Real Jardín Botánico (CSIC), Madrid.

Conway, V. M. 1936. Studies in the autoecology of Cladium mariscus R.Br. I: Structure and development. New Phytologist 35: 177-205. https://doi.org/10.1111/j.1469-8137.1936. tb06875.x
Conway, V. M. 1937. Studies in the autoecology of Cladium mariscus R.Br. II: The aeration of the subterranean parts of the plant. New Phytologist 36: 359-380. https://doi. org/10.1111/j.1469-8137.1937.tb06904.x

Conway, V. M. 1938a. Studies in the autoecology of Cladium mariscus R.Br. III: Growth rates of the leaves. New Phytologist 37: 254-278. https://doi.org/10.1111/j.1469-8137.1938. tb06939.x

Conway, V. M. 1938b. Studies in the autoecology of Cladium mariscus R.Br. IV: The distribution of the species. New Phytologist 37: 312-328. https://doi. org/10.1111/j.1469-8137.1938.tb06944.x

Coops, H. 2002. Ecology of charophytes: an introduction. Aquatic Botany 72: 205-208. https://doi.org/10.1016/ S0304-3770(01)00200-5

Den Hartog, C. \& Segal, S. 1964. A new classification of the water plant communities. Acta Botanica Neerlandica 13: 367393. https://doi.org/10.1111/j.1438-8677.1964.tb00163.x

DOCE (Diario Oficial de las Comunidades Europeas) 2000. Directiva 2000/60/CE del parlamento Europeo y del Consejo de 23 de octubre de 2000 por la que se establece un marco comunitario de actuación en el ámbito de la política de agua. Diario Oficial de las Comunidades Europeas L327(2000): 1-73.

Domínguez, F., Santisteban, J. I., Mediavilla, R. M., Dean, W. E., López, E., Gil, M. J. \& Ruiz, M. B. 2006. Cambios en el almacenamiento de $\mathrm{C}$ en el Parque Nacional de Las Tablas de Daimiel (PNTD) en los últimos 1000 años. Boletín Geológico y Minero 117: 537-544.

INYPSA 1990. Estudio de seguimiento de los resultados del Plan de Regeneración Hídrica del Parque Nacional de Las Tablas de Daimiel. INYPSA, Madrid.

Laguna, C., López-Perea, J. L., Viñuela, J. et al. 2016. Effects of invasive fish and quality of water and sediment on macrophytes biomass, and their consequences for the waterbird community of a Mediterranean floodplain. Science of the Total Environment 551-552: 513-521. https://doi. org/10.1016/j.scitotenv.2016.02.059

Ortiz Llorente, M. J. 2013. Sequía y ciclo del $\mathrm{CO}_{2}$ : Respuesta de las plantas emergentes en un humedal. Tesis Doctoral, Museo Nacional de Ciencias Naturales-CSIC, Madrid.

Ribeiro, M. D. 2004. Biología de los macrófitos emergentes en un humedal semiárido ibérico: Las Tablas de Daimiel. Tesis Doctoral, Universidad Complutense, Madrid.

Rodrigo, M. A., Alonso-Guillén, J. L. \& Soulié-Märsche, I. 2010. Reconstruction of the former charophyte community out of the fructifications identified in Albufera de València lagoon sediment. Aquatic Botany 92: 14-22. https://doi. org/10.1016/j.aquabot.2009.09.002

Sánchez Carrillo, S. \& Angeler, D. G. (Eds.) 2010. Ecology of threatened semi-arid wetlands. Springer, Dordrecht.

Van den Berg, M. S., Scheffer, M. \& Coops, H. 1998. The role of characean algae in the management of eutrophic shallow lakes. Journal of Phycology 34: 750-756. https://doi. org/10.1046/j.1529-8817.1998.340750.x 